\title{
Automatic Semiconductor Wafer Image Segmentation for Defect Detection Using Multilevel Thresholding
}

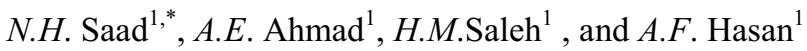 \\ ${ }^{1}$ School of Microelectronic Engineering, Universiti Malaysia Perlis, KampusPauh Putra, 02600 Arau, \\ Perlis, Malaysia
}

\begin{abstract}
Quality control is one of important process in semiconductor manufacturing. A lot of issues trying to be solved in semiconductor manufacturing industry regarding the rate of production with respect to time. In most semiconductor assemblies, a lot of wafers from various processes in semiconductor wafer manufacturing need to be inspected manually using human experts and this process required full concentration of the operators. This human inspection procedure, however, is time consuming and highly subjective. In order to overcome this problem, implementation of machine vision will be the best solution. This paper presents automatic defect segmentation of semiconductor wafer image based on multilevel thresholding algorithm which can be further adopted in machine vision system. In this work, the defect image which is in RGB image at first is converted to the gray scale image. Median filtering then is implemented to enhance the gray scale image. Then the modified multilevel thresholding algorithm is performed to the enhanced image. The algorithm worked in three main stages which are determination of the peak location of the histogram, segmentation the histogram between the peak and determination of first global minimum of histogram that correspond to the threshold value of the image. The proposed approach is being evaluated using defected wafer images. The experimental results shown that it can be used to segment the defect correctly and outperformed other thresholding technique such as Otsu and iterative thresholding.
\end{abstract}

\section{Introduction}

Semiconductor fabrication industry has greater competition where it is being influenced by factors of cost, yield, reliability and delivery time. Each process in semiconductor wafer fabrication becomes crucial including testing and quality control. In these processes, one of the main activities is defect detection. Defect detection involves imaging and analysis of the whole surface of the semiconductor wafer. The objective of this task is to provide an analysis of production batches, i.e. the numbers of good and defective wafers. Most of semiconductor manufacturer used human experts for doing the visual inspection process on the wafer surface to detect any defects [1]. By using human experts, the visual inspection

\footnotetext{
* Corresponding author: hidayahsaad@unimap.edu.my
} 
process needs supreme concentration and the time used to inspect each wafer is limited [2]. This process can be replaced by machine vision system. In order to be adopted into machine vision system, the wafer image can be segmented into background and the defect. In defect segmentation, we are only interested at the defected portion of the image.

Over the years, extensive researches have been done for semiconductor defect detection. A semiconductor defect detection using a novel fractal encoding algorithm has been proposed by Gleason et. al [3] that emphasized non-referential defect detection (NRDD). This research used new algorithmic rule that wrote of two major steps via the use of fractal images encoding and enhanced defect boundary delineation using active contours. Fractal image encoding broke the image to different sub regions and then determined the best set of affine transformations map one area to another.

Zontak et.al [4] suggested a defect detection procedure that avoid image registration and robust to variations of the pattern. The method used is based on anisotropic kernel reconstruction of the original image by using a reference image. The sources and reference images are mapped into a feature space, where each features are estimated by the weighted sum of neighbouring characteristics reference images. They shown that the features derived from the areas of disability cannot be reconstructed from the reference image and can be identified.

P.Bourgeat studied the wafer image segmentation by the Gabor filters [5]. He developed a technique designed to train the classifier with a small set of training samples and applied to wafer inspection optical tool known as Direct-to-Digital Holography (DDH) tool. The phase information shown a strong invariance to non uniformities and can readily be used for image segmentation.

Image segmentation involves subdividing the image into areas of similar attributes. It is one of the most crucial and challenging tasks in image analysis and pattern recognition. Image segmentation methods are generally based on one of two fundamental properties of the intensity values of image pixels: similarity and discontinuity [6]. Similarity concept is focusing on partitioning the image into several different regions and there must be similar image pixels that belong to a region according to a set of predefined criteria. Discontinuity concept will partition the image on the basis of abrupt changes in the intensity values of the image. Based on these two concepts, many segmentation methods have been introduced and can be divided into six main categories which are Edge-based, Threshold based, Region-based, Neural Network based, Cluster-based and Hybrid [7].

This paper presents an efficient image segmentation method using multilevel thresholding technique based on histogram segmentation. Using this method, it is possible to determine the threshold value effectively.

\subsection{Histogram thresholding}

Clustering is one of the methods that can be used for segmentation. A clustering technique classifies the objects into different groups, or more specifically, partitioning of a data set into clusters (subsets) [8]. By doing that, the data in each cluster ideally shares some common trait. The histogram of an image contains some peaks, so it can be separated into a number of modes. Each mode is expected to correspond to a region, and there exists a threshold at the valley between any two adjacent modes [9]. In this project, a histogram based thresholding technique proposed by Papamarkos\& Gatos known as multilevel thresholding is adopted to segment the semiconductor wafer defect from image background. In general, this technique can be divided into three main stages. At the first stage, a hill-clustering technique was applied to the image histogram in order to approximately determine the peaks location in the histogram [11]. Then, by using a linear mini-max approximation function, the histogram segments between the peaks were 
identified and approximated to a rational function [11]. Finally, the global minimum of each rational function was determined using one dimensional golden search minimization algorithm [10].

Consider that hill clustering gives a total number of histogram peaks equal to $P$, where $\left(W_{n}, G\left(W_{n}\right)\right), n=1, \ldots \ldots ., P$, are the coordinates of the peaks[10]. This means that the histogram has $P-1$ valleys which lie between the peaks. For the nth valley let:

- $K$ be the total number of gray levels included;

- $G\left(w_{k}\right), k=1, \ldots \ldots ., K$, be the values of the histogram at the $w_{k}$ gray level

- $w_{k}, k=1, \ldots \ldots ., K$, be the gray values with $W_{n} \leq w_{k} \leq W_{n+1}$

For each valley, the Linear Rational Approximation (LRA) algorithm can fit the histogram points $\left(w_{k}, G\left(w_{k}\right)\right), k=1, \ldots \ldots ., K$ by a real function of the general form [10]:

$$
R(w)=\frac{A(w)}{B(w)}=\frac{\sum_{m=0}^{N} a_{m} w^{m}}{1+\sum_{m=1}^{M} b_{m} w^{m}}
$$

where $a_{m}$ and $b_{m}$ are the unknown coefficients, $M$ and $N$ are integers that define the degree of the polynomials $A(w)$ and $B(w)$. Minimax criterion was used to solve this approximation problem.

The rational function $R(w)$ is real and continuous. Therefore, the one dimensional golden search algorithm was applied in order to find the minimum value in the region $W_{n}$, $W_{n+l}$. The inputs of the golden search algorithm are only the limits of the interval which defined by $W_{n}$ and $W_{n+1}$ while the one dimensional function for each region is the real rational function, $R(w)$. To ensure that the golden search algorithm always converges to the global minimum, the following procedures were used [10]:

Step 1: Find the minimum, $R_{\min }$ of $R(w)$.

Step 2 : Define the function $Y(w)$ according to the relation:

$$
Y(w)=\left\{\begin{array}{l}
R(w), \text { if } R(w) \leq R_{\min } \\
R_{\min }, \text { otherwise }
\end{array}\right.
$$

Step 3 : Find the minimum, $Y_{\min }$ of $Y(w)$.

Step 4 : If $Y_{\min }=R_{\min }$, go to step 5, otherwise put $R_{\min }=Y_{\min }$ and go step 2.

Step 5 : The global minimum solution is equal to $R_{\min }$ The procedure is terminated.

From the above procedure, the minimum value of $R(w)$ and its position was being determined. The minimum value of the first hill segment was taken as a threshold value of the histogram.

\subsection{Defect segmentation}

Fig. 1 shows the framework of automatic wafer image defect segmentation using multilevel thresholding. 


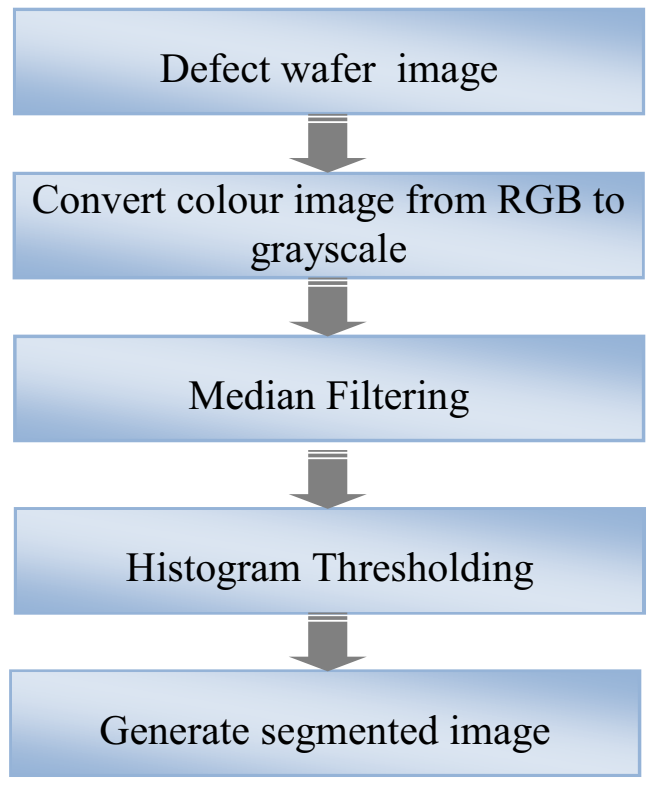

Fig. 1. Automatic Wafer Image Defect Segmentation.

Based on Fig. 1, the defect segmentation system operates by reading the input which is defected semiconductor wafer image in RGB image. Then, the RGB image was converted to gray scale image for simplifying the computational process. Each pixel in the gray scale image has intensity value from 0 (black) to 255 (white). The next step is to apply image filtering for modifying or enhancing an image to emphasize certain features or remove other features. In this project, median filtering was used to reduce the outliers without reducing the sharpness of image. The size of neighborhood used is $3 \times 3$. After completed enhancing the image, modified multilevel thresholding was used to find the threshold value for segmenting the defect from the background. The multilevel thresholding technique used histogram as a base to find the threshold value.

\section{Experimental results}

In order to demonstrate the performance of the proposed method, semiconductor wafer image has been used as an input. Three types of semiconductor wafer defect that commonly occur in the semiconductor industry have been chosen which are contamination, chip and crack. The images have been taken using high power microscope. The defects are shown by the black pixels in Fig. 2.

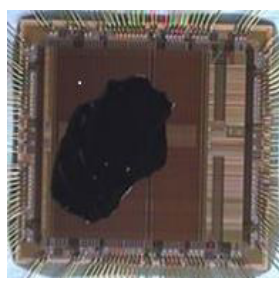

(a)

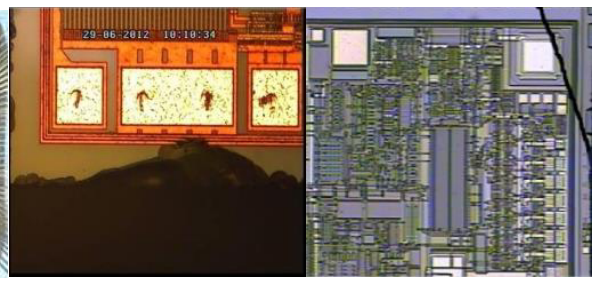

(c)

Fig. 2 Sample Wafer Images With (a) Contamination (b) Chip (c) Crack. 
Table 1 shows the results of each steps for the automatic defect segmentation using multilevel thresholding. There are three types of defects that have been used as an input to the proposed method which are contamination, chip and crack. By referring to the imagesafter multilevel thresholding, they show that the defect had successfully segmented from the background for all three types of defect. The defects are indicated by the black pixels while the background is indicated by the white pixel. The threshold values found from multilevel thresholding using hill clustering approach are 45, 43 and 7 for each type of input image.

Table 1. Results of Each Steps on Wafer Image Defect Segmentation Using Histogram Thresholding.

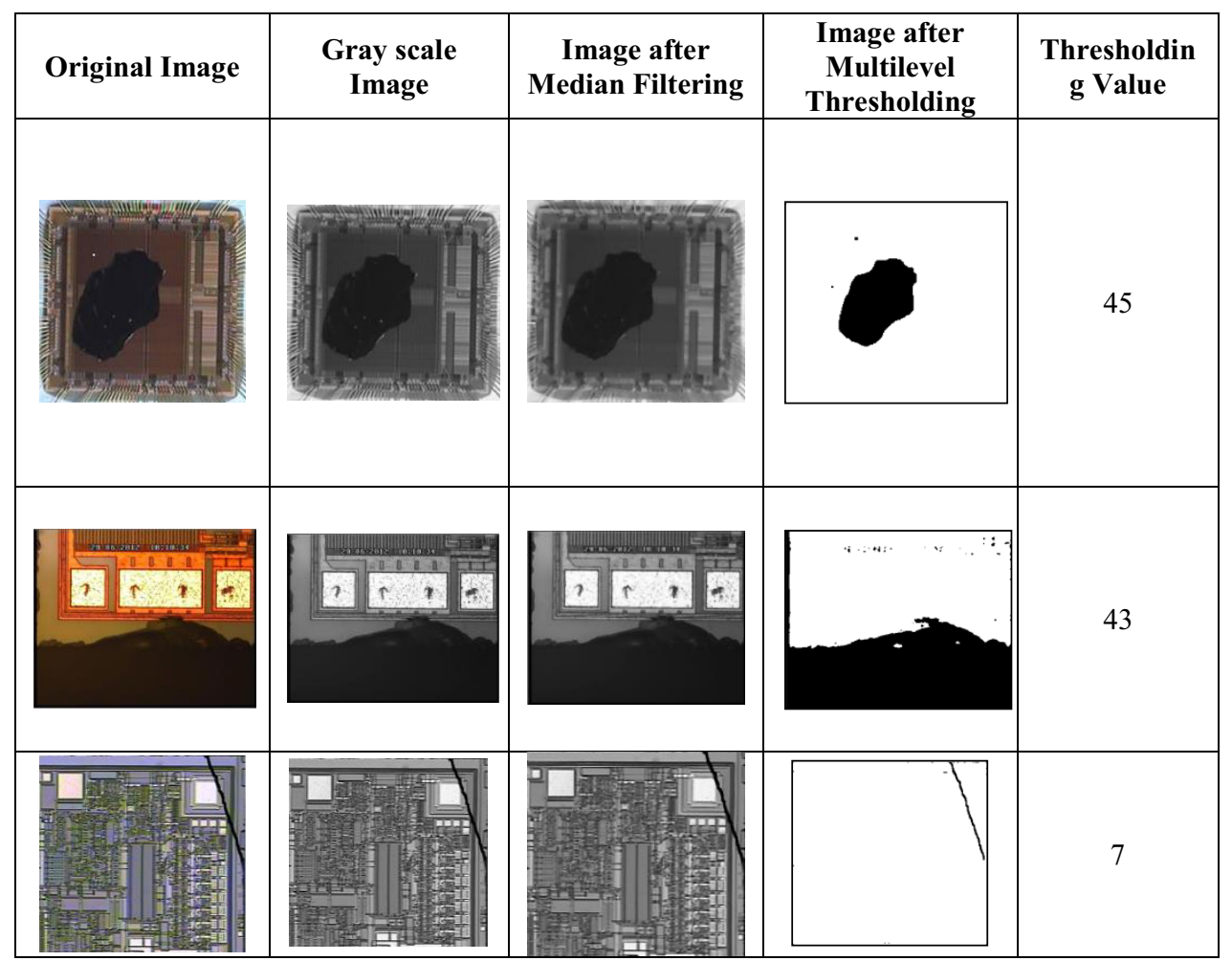

Table 2 shows the input image, Multilevelthresholding, Otsu thresholding and Iterative thresholding images. The threshold value computed by multilevel thresholding technique clearly segments the defect compared with other two techniques. Experimental result shows that multilevel thresholding outperforms the Otsu and Iterative thresholding in segmenting defect on semiconductor wafer image. This is due to Otsu and Iterative Thresholding techniques are more suited to the images that produce bimodal gray level histogram. 
Table 2. Comparison of Thresholding Image and Value Between Multilevel, Otsu, and Iterative Thresholding Technique.

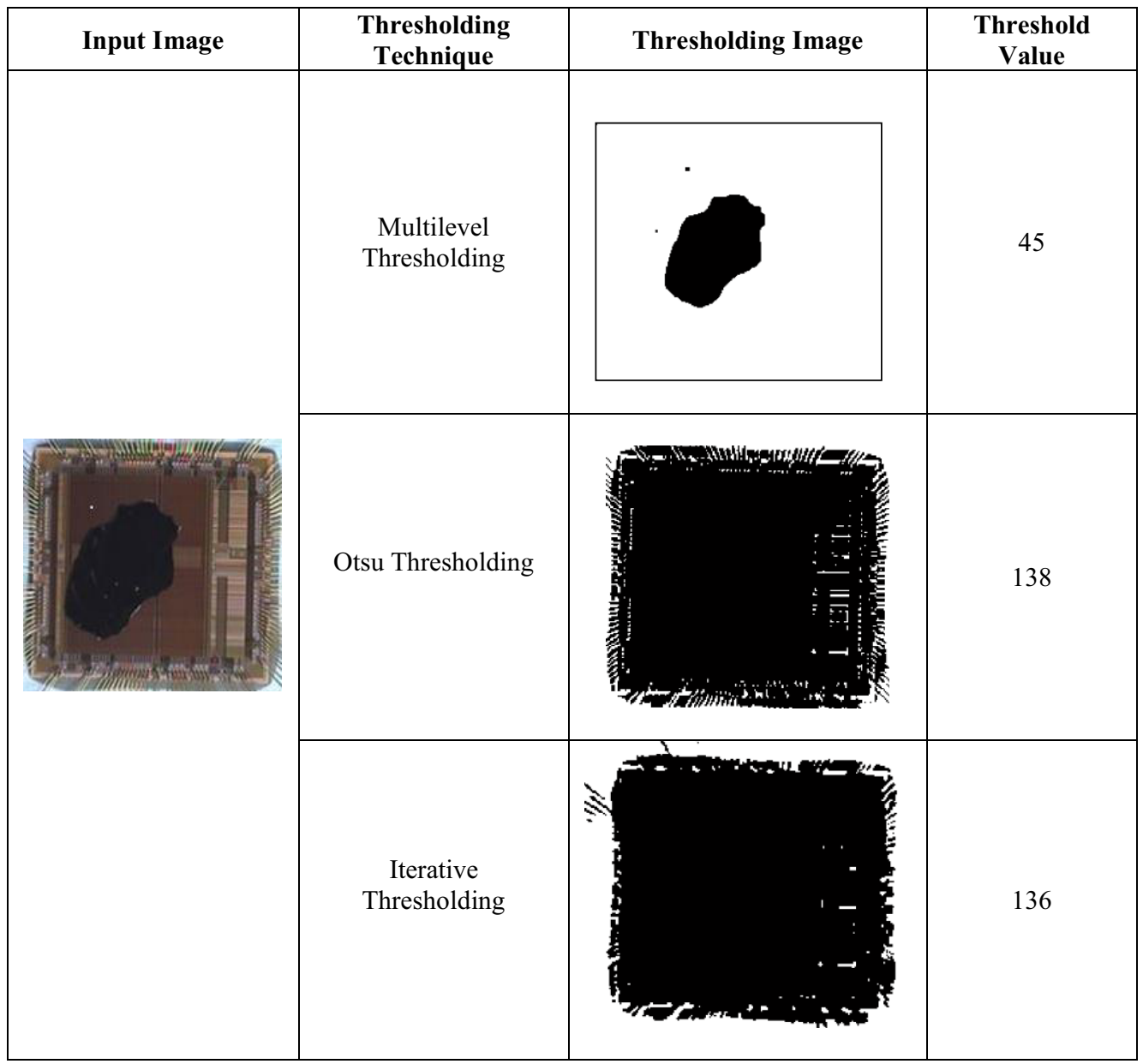

\section{Conclusions}

The automatic defect segmentation of the semiconductor wafer image is proposed and evaluated in this paper. The proposed approach used multilevel thresholding technique to determine the threshold value that further be used to segment the defect from image background. We have used three types of defected wafer images for the experimental observations and evaluation of the proposed method. Experimental results suggest that the proposed approach is able to segment the defected area from the background.

The authors would like to thank the staff from Microfabrication Laboratory, School of Microelectronic Engineering Universiti Malaysia Perlis for providing the sample of semiconductor wafer images. 


\section{References}

1. N.G. Shankar, Z.W. Zhong, Microelectronic Engineering, 77, 337 (2005)

2. Chen, Fei-Long, Shu-Fan Liu, IEEE transactions on semiconductor manufacturing, 13, $366(2000)$

3. S. Shaun Gleason, K. Regina Ferrell, P. Thomas Karnowski, W. Kenneth Tobin, Design, Process Integration, and Characterization for Microelectronics SPIE Proceeding, Santa Clara, CA, US (2002)

4. Zontak Maria, Israel Cohen, Machine Vision and Applications, 21, 129 (2010)

5. P. Bourgeat, F. Meriaudeau, Jr. Tobin, K.W. Gorria, International Society for Optics and Photonics (2004)

6. MacQueen, James, Proceedings of the $5^{\text {th }}$ Berkeley symposium on mathematical statistics and probability (1967)

7. Rajeshwar Dass, Priyanka, Swapna Devi, Image Segmentation Techniques, 1, (2012)

8. S.R. Dubey, P. Dixit, N. Singh, J.P. Gupta, IJIMAI, 2, 65 (2013)

9. Cheng Heng-Da, X.H. Jiang, Jingli Wang, Pattern recognition, 35, 373 (2002)

10. Papamarkos Nikos, Basilios Gatos, Graphical Models and Image Processing, 56, 357 (1994)

11. Tsai Du-Ming, Ying-Hsiung Chen, Pattern Recognition Letters, 13, 245 (1992) 